\title{
Self-Consistent Harmonic Oscillator Model and Tilted Rotation
}

\author{
W.D. Heiss ${ }^{\star}$ and R.G. Nazmitdinov ${ }^{\star, \star \star}$ \\ * Centre of Theoretical Physics and School of Physics \\ University of the Witwatersrand, PO Wits 2050, Johannesburg, South Africa \\ ** Bogoliubov Laboratory of Theoretical Physics, Joint Institute for Nuclear Research, 141980 Dubna, Russia
}

November 1, 2018

The three dimensional harmonic oscillator model including a cranking term is used for an energy variational calculation. Energy minima are found under variation of the three oscillator frequencies determining the shape of the system for given values of the three components of the rotational vector which determines the orientation of the angular momentum in the intrinsic frame. Tilted rotations are established by numerical means for triaxial nuclei. The onset of tilted rotations is related to an instability of the mean field as verified by the random phase approximation. Analytic expressions are derived for the critical rotational frequencies associated with the mean field instability.

PACS numbers: 21.60.Ev, 21.60.Jz, 21.10.Re, 03.75.Fi

\section{INTRODUCTION}

Numerous experimental observations of well developed rotational bands implying $\Delta I=1$ sequences (see for a review [1]) raise the question about mechanisms creating such collective states. The conventional description within the Cranking Shell Model (CSM) [2] suggests the $\Delta I=2$ sequence of rotational states. As is well known, in the CSM the axis of rotation coincides with the principal axis of the density distribution of the nucleus (principal axis cranking or PAC). As a result, the CSM Hamiltonian adheres to the $D_{2}$ spatial symmetry with respect to rotation by the angle $\pi$ around the rotational axis, for instance, the $x$ - axis. Consequently, all rotational states can be classified by the quantum number called signature $r=\exp (-i \pi \alpha)$ leading to selection rules for the total angular momentum $I=\alpha+2 n, n=0, \pm 1, \pm 2 \ldots$ In par- ticular, in even-even nuclei the lowest rotational (yrast) band characterised by the positive signature quantum number $r=1(\alpha=0)$ consists of even spins only.

It was suggested [3] that non-principal axis rotations which invalidate the concept of signature symmetry is responsible for rotational bands with $\Delta I=1$ sequences. This idea has led to considerable experimental and theoretical efforts during the past few years [1]. Related questions have been addressed recently in connection with effects of superfluidity in a trapped Bose-Einstein condensate (BEC) [1].

For a nucleus, the non-principal axis rotation is considered stationary. For the BEC, a precession of the symmetry axis is assumed for the condensate around the symmetry axis of the confining trap with the precession frequency being fixed by the angular momentum carried by the vortex line. A quantised vortex is aligned along the symmetry axis of the condensate carrying one unit $\hbar$ of angular momentum per particle. Similarly, the precessional motion of the principal axes in the nucleus creates vibrational (wobbling) excitations above the yrast line $[5[7$. Notice that in this case the wobbling excitations are quantum mechanical fluctuations of the angular momentum around a fixed principal axis of the mean field generated by the CSM $[6,7]$. These excitation carry one unit of angular momentum, similar to the vortex in the BEC, and corresponding states may be associated with odd spin partners of the gamma band in a rotating nucleus at low angular momenta [3]. It has been 
demonstrated [9] that at high rotational frequency the wobbling excitations become particularly low. Similar to the transition from spherical to deformed nuclei [5] when quadrupole excitation energies tend toward zero, an analogous mechanism is expected to prevail in that for vanishing wobbling excitations the nucleus undergoes a transition to tilted rotation as the energetically favoured state. The analysis of the link between tilted rotation and wobbling motion is one major aspect of the present paper.

In fact, a planar tilted rotation effecting the transition from the superfluid to the normal phase has been discussed within the phenomenological two-phase model [10]. The first microscopic attempt to describe a nonprincipal axis rotation has been carried out in [11] using the triaxial cranked oscillator model (TCO) with constant angular frequency (see for a review of latest studies [1]). However, the TCO calculations employ a fixed triaxial deformation. The violation of the self-consistency between rotation and evolution of shape parameters is a serious drawback of this model. In the present paper we investigate the occurrence of tilted rotation using the self-consistent triaxial cranked oscillator model. The model guarantees full self-consistency at all rotational frequencies and all particle numbers. A major outcome of the present paper is the result that, in even-even nuclei, tilted rotations occur if and only if the nucleus has a triaxial shape in its ground state. The pairing interaction could have an effect upon the conclusions drawn in the present paper. However, its role is less important at high spins where tilted rotations should be observed [1]. On a technical footing, it involves additional variational parameters which complicates the minimisation procedure. Therefore, we exclude in our model the pairing interaction. The transparency of the model which provides a general tendency of the phenomena and the succinct results compensate for this shortcoming.

In Sec.II we review the main features of our model. The numerical results are presented in Sec.III. The results are discussed in Sec.IV and a short summary in Sec.V. Technical details are deferred to Appendices. First preliminary results have been reported in 12,13.

\section{THE MODEL}

The many-body Hamiltonian (Routhian) in the rotating frame is given by

$$
H=\sum_{i=1}^{N}\left(h_{0}(i)-\vec{\Omega} \cdot \vec{l}(i)\right)=H_{0}-\vec{\Omega} \cdot \vec{L}
$$

where the single particle triaxial harmonic oscillator Hamiltonian $h_{0}$ is aligned along its principal axes and reads

$$
h_{0}=\frac{1}{2 m} \vec{p}^{2}+\frac{m}{2}\left(\omega_{x}^{2} x^{2}+\omega_{y}^{2} y^{2}+\omega_{z}^{2} z^{2}\right) .
$$

The rotational vector $\vec{\Omega}$ of the cranking term has the components $\left(\Omega_{x}, \Omega_{y}, \Omega_{z}\right)=\Omega(\sin \theta \cos \phi, \sin \theta \sin \phi, \cos \theta)$.

In Appendix A it is shown how this Hamiltonian is cast into the form

$$
H=\sum_{j=1}^{N} \sum_{k=1}^{3} E_{k}\left(Q_{k}^{\dagger} Q_{k}+1 / 2\right)_{j}
$$

where the normal mode energies $E_{k}$ are obtained from a cubic equation and the normal mode operators $Q_{k}$ are linear superpositions of the $p_{i}$ and $x_{i}$. In this way we have the exact single-particle energies and wave functions at our disposal. Note that, for $\Omega \neq 0$ and in particular for a tilted rotation, the normal mode operators $Q_{k}$ are superpositions of all three Cartesian components of the 
$p_{i}$ and $x_{i}$. Furthermore, the eigenmode energies $E_{k}$ are real only for a certain range of $\Omega \leq \Omega_{\max }$ (see Appendix B). We will restrict ourselves to this physical range of values of the rotational frequency.

The knowledge of the eigenmodes and eigenfunctions enables us to calculate energy contours of the total energy

$$
E_{\mathrm{tot}}=E_{1} \Sigma_{1}+E_{2} \Sigma_{2}+E_{3} \Sigma_{3}
$$

in, say, the $\omega_{x}-\omega_{y}$ plane with the particle number $N$ and the rotational vector $\vec{\Omega}$ as parameters. The configurations are determined by $\Sigma_{k}=\Sigma_{j}^{N}\left(n_{k}+1 / 2\right)_{j}$ where the occupation numbers $n_{k}$ are the eigenvalues of $Q_{k}^{\dagger} Q_{k}$, they take the values $0,1,2, \ldots$ For the PAC rotation around the $x$-axis the notation $\Sigma_{1} \equiv \Sigma_{x}, \Sigma_{2} \equiv \Sigma_{+}, \Sigma_{3} \equiv \Sigma_{-}$is used 18] (we chose $\omega_{x} \geq \omega_{y} \geq \omega_{z}$, see also Appendix B).

The ground state is determined by filling the singleparticle levels from the bottom. We take care of the particle spin only in obeying the Pauli principle which allows two particles in one level; also we consider only one kind of nucleons, protons or neutrons. It is clear that different sets of normal modes yield different sets of occupation numbers. A pronounced shell structure can exist only for special sets of normal modes. Correspondingly, all spherical shells will be strongly mixed in our model, in particular the $\Delta N=2$ mixing is taken into account exactly. In this way we go beyond the calculation of [2,19] where mixing between major oscillator shells is ignored.

The normal modes depend on the three components of the rotational vector and on the harmonic oscillator frequencies. From our assumption that the system adjusts itself under the influence of the rotation by minimising $E_{\text {tot }}$, a change of the magnitude or direction of the rotation leads to a corresponding change of the effective mean field potential which is given by the oscillator frequencies. In other words, for a given rotational frequency, we must seek the minimum of $E_{\text {tot }}$ under variation of the oscillator frequencies. The variation cannot be unrestricted as the confining potential encloses a fixed number of particles, and assuming that the particle density does not change we are led to a fixed volume constraint

$$
\omega_{x} \omega_{y} \omega_{z}=\omega_{0}^{3}=(41)^{3} / N
$$

The volume constant is chosen in units where frequencies are given in $\mathrm{MeV}$ and lengths in Fermi. Introducing the Lagrange multiplier $\lambda$, we solve the variational problem

$$
\delta\left(\langle g|H| g\rangle-\lambda \omega_{x} \omega_{y} \omega_{z}\right)=0
$$

where $|g\rangle$ denotes the ground state as described above.

From Eq.(6) we obtain, after differentiation with respect to the oscillator frequencies and using Feynman's theorem [20]

$$
\frac{d}{d \omega_{k}}\langle g|H| g\rangle=\left\langle g\left|\frac{d H}{d \omega_{k}}\right| g\right\rangle,
$$

the self-consistency condition

$$
\omega_{x}^{2}\left\langle g\left|x^{2}\right| g\right\rangle=\omega_{y}^{2}\left\langle g\left|y^{2}\right| g\right\rangle=\omega_{z}^{2}\left\langle g\left|z^{2}\right| g\right\rangle
$$

which must be obeyed at the minimum of $E_{\text {tot }}$. Notice, that Eq.(8) becomes a general result which includes as a particular case the cranking harmonic oscillator with the PAC rotation around the $x$-axis 18 . The condition serves as an acid numerical test. Note that it is fulfilled irrespective of the minimum being local or global.

For $\Omega=0$ we recover the well known magic numbers of the triaxial harmonic oscillator for one type of particles (proton or neutron) [5] at $N=20,40,70,112, \ldots$ which manifest themselves in our procedure by the spherical symmetric solutions $\omega_{x}=\omega_{y}=\omega_{z}=\omega_{0}$. Between these 
magic numbers the well known prolate or triaxial near prolate and oblate or triaxial near oblate solutions are found, where a (near) prolate solution $\left(\omega_{x} \simeq \omega_{y}>\omega_{z}\right)$ is obtained for a shell which is less than half completed and a (near) oblate solution $\left(\omega_{x} \simeq \omega_{y}<\omega_{z}\right)$ for a more than half completed shell. In Fig.1 the values of $\left\langle x^{2}\right\rangle,\left\langle y^{2}\right\rangle$ and $\left\langle z^{2}\right\rangle$ are plotted versus $N$. From the figure we see, that, for $\Omega=0$, some shapes are axially symmetric while others are not. Switching on the rotation $\Omega \neq 0$ a similar picture is obtained as long as the rotational frequency is kept at a reasonably low value $(\Omega \leq 1.0$ for $N \leq 24$ and $\Omega \leq 0.3$ for $N \geq 40$ ). The corresponding minima of the total Routhian are a function of $\vec{\Omega}$, i.e. of $\Omega, \theta$ and $\phi$. Our interest is focused upon the lowest minimum under variation of $\theta$ and $\phi$ for fixed $\Omega$, i.e. $\partial E_{\text {tot }} / \partial \theta=$ $\partial E_{\mathrm{tot}} / \partial \phi=0$. If such minimum is found for $\theta \neq 0$ we refer to this solution as to a tilted solution.

\section{NUMERICAL RESULTS}

As is well known [18], the PAC rotation of prolate systems leads to triaxial shapes. With increasing rotational speed the change of the shape leads eventually, for a critical frequency $\Omega_{\text {crit }}^{(1)}$, to an oblate shape with the rotational axis coinciding with the symmetry axis. Using the minimising procedure of our model confirms this fact in principle. However, our model allows an easy assessment as to whether the minimum is local or global. It turns out that, for $N<56$, the minimum found at $\Omega_{\text {crit }}^{(1)}$ is no longer a global minimum, since somewhere in the interval $\left[0, \Omega_{\text {crit }}^{(1)}\right]$ another lower lying minimum comes up. As a typical example we consider the celebrated nucleus ${ }^{20} \mathrm{Ne}$ (since we take into account one kind of particle, this nucleus corresponds to system with ten particles). For $\Omega=0$ the global minimum occurs for a prolate shape with the configuration $\left(\Sigma_{1}, \Sigma_{2}, \Sigma_{3}\right)=(7,7,11)$. With increasing $\Omega$ the minimum initially continues to be global and the configuration remains constant while the nucleus becomes triaxial. However, for $\Omega \approx 4.55$ another lower minimum occurs with a completely different, probably non-physical configuration $\left(\left(\Sigma_{1}, \Sigma_{2}, \Sigma_{3}\right)=(5,5,25)\right)$. Only if the original configuration is enforced, the oblate shape occurs at the larger value $\Omega_{\text {crit }}^{(1)} \approx 5.65$, but, being a local minimum, it may have to be interpreted as an isomeric state.
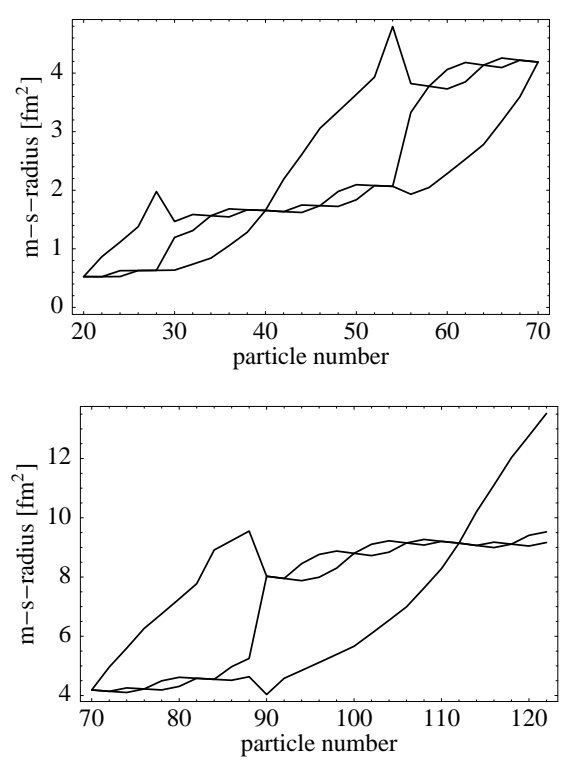

FIG. 1. Mean square radii $\left\langle x^{2}\right\rangle,\left\langle y^{2}\right\rangle$ and $\left\langle z^{2}\right\rangle$ versus particle number for minimal energy configurations without rotation in the triaxial harmonic oscillator. The magic (spherical), axially symmetric and triaxial configurations are clearly discernible.

The observation, that the onset of an oblate shape is attained at a local and not at a global minimum, prevails for all prolate nuclei with $N \leq 56$. When looking at Fig.1 there are the nuclei which have, for $\Omega=0$, a triaxial shape close to the prolate shape, for instance $N=22,44,48,50$. Initially when $\Omega$ is switched on, they behave just like the case considered in the previ- 
ous paragraph in that they reach eventually, at $\Omega_{\text {crit }}^{(1)}$, the oblate shape at a local minimum. For a certain value $\Omega_{\text {crit }}^{(2)}>\Omega_{\text {crit }}^{(1)}$ the onset of tilted rotation arises. In other words, enforcing the initial configuration and thus pertaining to the local minimum, the minimum is now found at $\theta>0$ and possibly $\phi>0$ while the minimum prevailing within the interval $\left[\Omega_{\text {crit }}^{(1)}, \Omega_{\text {crit }}^{(2)}\right]$ for $\theta=0$ has, at $\Omega=\Omega_{\text {crit }}^{(2)}$, become unstable, in fact it turns into a maximum in the $\theta-\phi$ plane. In the following section we derive analytic expressions for $\Omega_{\text {crit }}^{(2)}$ and $\Omega_{\text {crit }}^{(1)}$, and provide an explanation why the tilted rotation cannot occur when starting with a prolate nucleus irrespective of the minimum being local or global. In this context we stress that the model has of course its limitations and must be used with due discretion. The occurrence of new global minima for increasing values of $\Omega$ is an expected feature of the model which does not necessarily have a physical significance. For light prolate nuclei, where the onset of oblate shapes occurs at values of $\Omega$ so large that the minimum is not global, only a more sophisticated approach could ascertain as to whether such a minimum is stable. Likewise, there is a temptation to associate the new global minimum with fission, but this also lies beyond the scope of the present paper.

In contrast, for all oblate (and heavy prolate nuclei) the minima at $\Omega_{\text {crit }}^{(1)}$ are global as the critical values are sufficiently small. In these cases we therefore view our findings as reliable and physically significant. As is shown in the following section analytically, tilted solutions can occur at $\Omega_{\text {crit }}^{(2)}>\Omega_{\text {crit }}^{(1)}$ and, in fact do occur, when starting from a nucleus that is triaxial for $\Omega=0$. In turn, when starting from an oblate nucleus, a tilted solution never occurs. Yet, the pattern is very similar in both cases for
$\Omega<\Omega_{\text {crit }}^{(1)}$, in that once the nucleus has become triaxial for $\Omega>0$, it attains the oblate shape for $\Omega=\Omega_{\text {crit }}^{(1)}$, where, for the energy minimum, the rotational axis coincides with the symmetry axis.

\section{BIFURCATION POINTS}

The first subsection is devoted to the basics of our approach with particular emphasis on the extension of established knowledge. In the following subsections the calculation and the role of critical frequencies are discussed.

\section{A. Basics}

The two critical frequencies, $\Omega_{\text {crit }}^{(1)}$ and $\Omega_{\text {crit }}^{(2)}$, are closely related to an instability of the mean field. When $\Omega$ is sweeping beyond the critical value, the mean field changes and may lead to a symmetry breaking. The instability can be identified as a bifurcation point 21] where new solutions that break the symmetry emerge. As is demonstrated below, they are related to collective vibrations carrying nonzero angular momentum aligned along the symmetry axis. The bifurcation points are defined by the vanishing vibrational frequencies in the rotating frame. That occurs at a discrete set of cranking frequencies given by

$$
\Omega_{\mathrm{crit}} \equiv \omega_{K} / K
$$

where $K$ is the angular momentum along the symmetry axis carried by a collective vibration with the energy $\omega_{K}$ in the laboratory frame.

We treat the collective excitations in the random phase approximation (RPA). The variation in the one-body potential around the equilibrium shape determines the ef- 
fective quadrupole-quadrupole interaction [22]. As a result, the total Hamiltonian can be presented as

$$
H_{\mathrm{RPA}}=H_{0}-\vec{\Omega} \cdot \vec{L}-\frac{\kappa}{2} \sum_{\mu=-2}^{2} D_{\mu}^{\dagger} D_{\mu}=\tilde{H}-\vec{\Omega} \cdot \vec{L}
$$

where the quadrupole operators $D_{\mu}=r^{2} Y_{2 \mu}$ are expressed in terms of the double-stretched coordinates $\bar{q}_{i}=$ $\frac{\omega_{i}}{\omega_{0}} q_{i},\left(q_{i}=x, y, z\right)$ (see also Appendix C). Note that the effective interaction restores the rotational invariance of the Hamiltonian $H_{0}$ in that now $\left[\tilde{H}, L_{i}\right]=0 \quad(i=$ $x, y, z)$. Also note that the self-consistency conditions Eq.(8) fix the quadrupole strength $\kappa=\frac{4 \pi}{5} \frac{m \omega_{0}^{2}}{\left\langle r^{2}\right\rangle}$, where $<r^{2}>=<\bar{x}^{2}+\bar{y}^{2}+\bar{z}^{2}>$.

We solve the RPA equation of motion for generalized coordinates $\mathcal{X}_{\lambda}$ and momenta $\mathcal{P}_{\lambda}$ (see for details 23):

$$
\begin{aligned}
{\left[H_{\mathrm{RPA}}, \mathcal{X}_{\lambda}\right] } & =-i \omega_{\lambda} \mathcal{P}_{\lambda}, \quad\left[H_{\mathrm{RPA}}, \mathcal{P}_{\lambda}\right]=i \omega_{\lambda} \mathcal{X}_{\lambda} \\
{\left[\mathcal{X}_{\lambda}, \mathcal{P}_{\lambda}\right] } & =i \delta_{\lambda, \lambda^{\prime}}
\end{aligned}
$$

Here, $\omega_{\lambda}$ is the RPA eigenfrequency in the rotating frame and the associated phonon operator is $O_{\lambda}=$ $\left(\mathcal{X}_{\lambda}-i \mathcal{P}_{\lambda}\right) / \sqrt{2}$. In contrast to the CRPA approach [6, 76,23] developed for the PAC rotation, the phonon is in the present model a superposition of different signature phonons. The degree of the mixture depends on the tilted angle: the signature and $|K|$ are good quantum numbers, respectively, for rotations coinciding with one of the principal axes. The solution of the RPA equations leads to the secular equation $F\left(\omega_{\lambda}\right)=0$ with $F$ being a five by five determinant. The roots are the RPA eigenfrequencies $\omega_{\lambda_{i}}$. The non-zero solutions appear in pairs $\pm \hbar \omega_{\lambda}$, we choose solutions with positive norm. The function $F$ factors into positive and negative signature parts for PAC rotations, since the signature is then a good quantum number. This simplifies the calculations of the RPA modes and provides the analytical solutions and the critical frequencies $\Omega_{\text {crit }}^{(1)}$ and $\Omega_{\text {crit }}^{(2)}$, where the minimal solution corresponds to the PAC rotation around the symmetry axis being the $x$-axis.

Since at this transition point the minimal solution corresponds to a one-dimensional rotation $(\vec{\Omega} \equiv(\Omega, 0,0))$ around the symmetry axis, the angular momentum is a good quantum number. The RPA states are characterised by the projection of the angular momentum upon the symmetry axis ( $x$-axis) because $\left[L_{x}, O_{\lambda}^{\dagger}\right]=\lambda O_{\lambda}^{\dagger}$ with $\lambda$ being the value of the angular momentum carried by the phonons along the $x$-axis. We thus obtain

$$
\begin{aligned}
{\left[H_{\mathrm{RPA}}, O_{\lambda}^{\dagger}\right]=} & {\left[\tilde{H}-\Omega L_{x}, O_{\lambda}^{\dagger}\right]=} \\
& \left(\tilde{\omega}_{\lambda}-\lambda \Omega\right) O_{\lambda}^{\dagger} \equiv \omega_{\lambda} O_{\lambda}^{\dagger} .
\end{aligned}
$$

This equation implies that at the rotational frequency $\Omega_{c r}=\tilde{\omega}_{\lambda} / \lambda$ one of the RPA frequency vanishes.

\section{B. Critical frequencies for oblate rotation}

\section{Mean field}

Let us first turn to our numerical findings relating to oblate and/or near oblate cases. In these cases the first critical rotational frequency is associated with a onedimensional rotation. We use $\Sigma_{1}<\Sigma_{2}<\Sigma_{3}$. Exploiting that at the transition point $\omega_{y}=\omega_{z}=\omega_{\perp}$, we obtain from Eq.(8) a third order equation for $u=\Omega / \omega_{\perp}$ which reads (see also [18])

$$
u^{3}-\frac{u}{2}+\frac{1}{2} \frac{r-1}{r+1}=0
$$

and $r=\Sigma_{3} / \Sigma_{2}$. From the discriminant of Eq.(13) we obtain the critical value

$$
r_{c r}=\frac{\sqrt{27}+\sqrt{2}}{\sqrt{27}-\sqrt{2}}
$$

It was shown in 18 that a prolate system becomes eventually oblate with the rotational axis coinciding with the 
symmetry axis, if $r<r_{c r}$. In general, for $r<r_{c r}$, we have the three solutions for Eq.(13)

$$
\begin{gathered}
u_{1}=\sqrt{\frac{2}{3}} \cos \frac{\chi}{3}, \quad u_{2,3}=-\frac{1}{2} \sqrt{\frac{2}{3}}\left(\cos \frac{\chi}{3} \mp \sqrt{3} \sin \frac{\chi}{3}\right) \\
\cos \chi=-3 \sqrt{\frac{3}{2}} \frac{r-1}{r+1}, \quad \pi / 2<\chi<\pi
\end{gathered}
$$

By their derivation these values correspond to three bifurcation points. We recall that for one-dimensional rotation the mean field equation Eq. 13. determines the positive signature solutions. At the left and right hand boundary values of the angle $\chi$ we obtain from Eqs.(15), (16)

$$
\begin{aligned}
\chi=\pi / 2 \quad(r=1) \Rightarrow\left\{\begin{array}{l}
\Omega_{1}=\omega_{\perp} / \sqrt{2} \\
\Omega_{2}=0 \\
\Omega_{3}=-\omega_{\perp} / \sqrt{2}
\end{array}\right. \\
\chi=\pi \quad\left(r=r_{\mathrm{cr}}\right) \Rightarrow\left\{\begin{array}{l}
\Omega_{1}=\sqrt{2 / 3} \omega_{\perp} / 2 \\
\Omega_{2}=\sqrt{2 / 3} \omega_{\perp} / 2 \\
\Omega_{3}=-\sqrt{2 / 3} \omega_{\perp}
\end{array}\right.
\end{aligned}
$$

The natural question arises: what is the meaning of the negative rotational frequencies? Below we demonstrate that the second solution gives the critical point $\Omega_{c r}^{(1)}$ at which the lowest positive signature vibrational frequency (in the rotating frame) tends to zero.

It should be noted that the solutions $\Omega_{2,3}\left(u_{2,3}\right)$ have been found in [18], however, an interpretation was not provided due to the lack of the RPA analysis. In 24 and 25] vibrational excitations for one-dimensional rotations around the $x$-axis have been dealt with by the RPA. However, in 24] the self-consistent condition and the residual interaction are different from those of the present paper, and in 25 the RPA analysis was done in the laboratory frame. We emphasise that it is important to treat the $\mathrm{RPA}$ equations in the rotating frame, since only then the relation between the bifurcation points of the mean field and the RPA solutions can be clearly worked out. This relation has not been analysed in the quoted papers.

\section{Positive signature solutions}

As noted above, the determinant $F\left(\omega_{\lambda}\right)$ factors into positive and negative signature blocks for the oblate rotation. With the projection $\lambda$ on the symmetry axis $x$ of the angular momentum $L_{x}$ being a good quantum number the phonon states with different $\lambda$ decouple. Therefore the secular equation for the positive signature phonons separates into three independent equations each determining the solution for the collective excitations with $\lambda=0$ and $\lambda= \pm 2$. We focus our analysis on the positive signature excitations with $\lambda= \pm 2$, since they will define the critical rotational frequency.

Taking into account the self-consistent condition (8) the secular equation can, after some tedious algebra, be presented for $\lambda=+2$ in the form

$$
\frac{a}{2+x}+\frac{b}{2-x}+\frac{c}{x}=1
$$

using the notation

$$
x=\frac{\omega_{\lambda}+2 \Omega}{\omega_{\perp}}, \quad a=\frac{1}{r+1}, \quad b=\frac{r}{r+1}, \quad c=\frac{r-1}{r+1} .
$$

The RPA eigen-frequencies $\omega_{\lambda}$ in the rotating frame are obtained by solving the cubic equation

$$
x^{3}-2 x+4 c=0
$$

leading to the solution

$$
\begin{aligned}
& \omega_{\lambda=+2}^{(1)}=2 \omega_{\perp} \sqrt{\frac{2}{3}} \cos \frac{\chi}{3}-2 \Omega \\
& \omega_{\lambda=+2}^{(2,3)}=-\omega_{\perp} \sqrt{\frac{2}{3}}\left(\cos \frac{\chi}{3} \mp \sqrt{3} \sin \frac{\chi}{3}\right)-2 \Omega .
\end{aligned}
$$

We consider only the first solution as it is the one with a positive norm. Setting this frequency equal to zero 
we find just the first bifurcation point $u_{1}=\Omega_{1} / \omega_{\perp}$ of Eq.(13)

$$
\Omega_{1}=\omega_{\perp} \sqrt{\frac{2}{3}} \cos \frac{\chi}{3}=\frac{\tilde{\omega}_{\lambda=+2}^{(1)}}{\lambda}
$$

where

$$
\tilde{\omega}_{\lambda=+2}^{(1)}=2 \omega_{\perp} \sqrt{\frac{2}{3}} \cos \frac{\chi}{3} .
$$

For $\chi=\pi / 2\left(r=\Sigma_{3} / \Sigma_{2}=1\right)$ we obtain from Eq.(25) the well known estimate of the isoscalar giant quadrupole resonance energy in non-rotating axially deformed nuclei, i.e. $\tilde{\omega}_{\lambda=+2}^{(1)}=\sqrt{2} \omega_{\perp}$ [26] with $\omega_{\perp}$ being the confinement frequency perpendicular to the symmetry axis of the mean field potential. The value $\chi=\pi$ yields $\tilde{\omega}_{\lambda=+2}^{(1)}=\sqrt{2 / 3} \omega_{\perp}$. For a fixed configuration $\Sigma_{1}<\Sigma_{2}<\Sigma_{3}$ the oblate state has the maximal angular momentum $L_{x}=\Sigma_{3}-\Sigma_{2}$ (ground-band termination state) [2]. If the signature symmetry is conserved the mode $\omega_{\lambda=+2}^{(1)}$ can make a transition to a different configuration with two more units of angular momentum than that of the oblate (yrast) state, assuming that the rotational frequency exceeds the critical frequency $\Omega_{1}$. Therefore, for a fixed configuration the first bifurcation point is related to the quadrupole vibrational mode $\tilde{\omega}_{\lambda=+2}^{(1)}$ in the laboratory frame carrying two units $(\lambda=+2)$ of angular momentum.

For the $\lambda=-2$ excitations the secular equation is similar to that of Eq.(19) with $x$ replaced by $-y$ where

$$
y=\frac{\omega_{\lambda}-2 \Omega}{\omega_{\perp}} .
$$

The RPA eigen-frequencies $\omega_{\lambda}$ are obtained by solving the cubic equation for $y$

$$
y^{3}-2 y-4 c=0
$$

leading this time to the solutions

$$
\begin{aligned}
& \omega_{\lambda=-2}^{(1,3)}=2 \Omega+\omega_{\perp} \sqrt{\frac{2}{3}}\left(\cos \frac{\chi}{3} \pm \sqrt{3} \sin \frac{\chi}{3}\right) \\
& \omega_{\lambda=-2}^{(2)}=2 \Omega-2 \omega_{\perp} \sqrt{\frac{2}{3}} \cos \frac{\chi}{3} .
\end{aligned}
$$

The positive norm solutions are the first and the third. Setting $\omega_{\lambda=-2}^{(1,3)}$ equal to zero, we obtain the second and third bifurcation points $u_{2,3}=\Omega_{2,3} / \omega_{\perp}$ of Eq.(13) which read

$$
\begin{aligned}
& \Omega_{2}=-\frac{\omega_{\perp}}{2} \sqrt{\frac{2}{3}}\left(\cos \frac{\chi}{3}-\sqrt{3} \sin \frac{\chi}{3}\right)=\frac{\tilde{\omega}_{\lambda=-2}^{(3)}}{\lambda} \\
& \Omega_{3}=-\frac{\omega_{\perp}}{2} \sqrt{\frac{2}{3}}\left(\cos \frac{\chi}{3}+\sqrt{3} \sin \frac{\chi}{3}\right)=\frac{\tilde{\omega}_{\lambda=-2}^{(1)}}{\lambda} \\
& \tilde{\omega}_{\lambda=-2}^{(1,3)}=\omega_{\perp} \sqrt{\frac{2}{3}}\left(\cos \frac{\chi}{3} \pm \sqrt{3} \sin \frac{\chi}{3}\right) .
\end{aligned}
$$

The frequencies $\tilde{\omega}_{\lambda=-2}^{(1,3)}$ are the vibrational modes in the laboratory frame carrying $\lambda=-2$ units of angular momentum.

A collective vibration with the projection of $K \neq 0$ units of angular momentum along the symmetry axis may be associated with a surface wave travelling around this axis. This wave can travel in opposite direction (negative rotational frequency) with respect to the rotation of the core (the vacuum) [21].

With $\chi=\pi / 2\left(\Sigma_{3}=\Sigma_{2}\right)$ we obtain $\tilde{\omega}_{\lambda=-2}^{(1)}=\sqrt{2} \omega_{\perp}=$ $\tilde{\omega}_{\lambda=+2}^{(1)}$ and $\tilde{\omega}_{\lambda=-2}^{(3)}=0$. In this case the angular momentum of the vacuum (oblate) state is zero, since $L_{x}=$ $\Sigma_{3}-\Sigma_{2}=0$. Thus, in the oblate system with zero angular momentum a breaking of the symmetry arises at the positive/negative rotational frequency

$$
\Omega_{\mathrm{cr}}=\tilde{\omega}_{\lambda= \pm 2}^{(1)} / \lambda= \pm \omega_{\perp} / \sqrt{2}
$$

It is at this point where the transition from non-collective rotation (around $x$-axis) to triaxial collective rotation takes place, where the latter state has two units of angular momentum. 
At the other end of the parameter range, $\chi=\pi(r=$ $\left.r_{c r}\right)$, we obtain $\tilde{\omega}_{\lambda=-2}^{(1)}=2 \sqrt{2 / 3} \omega_{\perp}$ and $\tilde{\omega}_{\lambda=-2}^{(3)}=$ $-\sqrt{2 / 3} \omega_{\perp}$. Within the full range of parameter values $\pi / 2<\chi<\pi\left(1<r<r_{\mathrm{cr}}\right)$ the vibrational modes $\tilde{\omega}_{\lambda=-2}^{(1,3)}$ are sweeping over the values

$$
\begin{aligned}
\sqrt{2} \omega_{\perp} & <\tilde{\omega}_{\lambda=-2}^{(1)}<2 \sqrt{\frac{2}{3}} \omega_{\perp}, \\
-\sqrt{\frac{2}{3}} \omega_{\perp} & <\tilde{\omega}_{\lambda=-2}^{(3)}<0 .
\end{aligned}
$$

When the rotational frequency approaches the lowest critical value for a triaxial nucleus which is

$$
\Omega_{2}=-\frac{\omega_{\perp}}{2} \sqrt{\frac{2}{3}}\left(\cos \frac{\chi}{3}-\sqrt{3} \sin \frac{\chi}{3}\right)=\Omega_{\mathrm{crit}}^{(1)}>0
$$

the vibrational excitation in the rotating frame for the oblate state tends to zero, i.e., $\omega_{\lambda=-2}^{(3)}=\tilde{\omega}_{\lambda=-2}^{(3)}+$ $\left.2 \Omega\right|_{\Omega=\Omega_{2}}=-\left|\tilde{\omega}_{\lambda=-2}^{(3)}\right|+2 \Omega_{\text {crit }}^{(1)}=0$. The negative quadrupole mode energy $\omega_{\lambda=-2}^{(3)}$ vanishes at the transition from the non-collective to the collective triaxial rotation and becomes positive for the oblate state at higher rotational frequencies of the mean field. In other words, the mode $\omega_{\lambda=-2}^{(3)}$ can be viewed as a de-excitation in the intrinsic frame of the vacuum (oblate) state thus leading to the triaxial state with two units of angular momentum less than the vacuum state. In contrast, the mode $\omega_{\lambda=-2}^{(1)}$ is positive at $\Omega_{\text {crit }}^{(1)}$ and describes an ordinary quadrupole excitation with two units of angular momentum less than that of the yrast state on which it is based.

\section{Negative signature solutions}

From the previous subsection we have learned that the critical rotational frequency is related to the vanishing of the RPA solutions in the intrinsic frame and consequently to the breaking of the mean field symmetry. Since the negative signature solutions are associated with the $\lambda= \pm 1$ excitations, at least one of the solutions must be related to signature symmetry breaking, i.e. to the bifurcation point where tilted rotation is setting in.

In the oblate case the secular equation for the negative signature excitations contains the "spurious" solution at $\omega=\Omega$ corresponding to a collective rotation and the vibrational modes; the latter include the wobbling motion and carry one unit of angular momentum [6,7]. Taking into account the self-consistent condition (8) the secular equation for the $\lambda=-1$ excitation reads

$$
\begin{aligned}
& \frac{e}{\omega_{x}+\omega_{\perp}+\Omega-x}+\frac{f}{\omega_{x}+\omega_{\perp}-\Omega+x} \\
& +\frac{d}{\omega_{x}-\omega_{\perp}-\Omega+x}+\frac{p}{\omega_{x}-\omega_{\perp}+\Omega-x}=\frac{2}{\omega_{x}}
\end{aligned}
$$

where we use the notation

$$
\begin{aligned}
e=\frac{1+k}{r+1}, & f=\frac{r+k}{r+1}, \\
d=\frac{1-k}{r+1}, & p=\frac{r-k}{r+1}, \quad k=\Sigma_{1} / \Sigma_{2} .
\end{aligned}
$$

The root in $x$ of Eq. 37 is the RPA frequency $\omega_{\lambda}$. After some simplification we obtain

$$
y\left(y^{3}-y-2 \alpha c\right)=0,
$$

where

$$
y=\frac{\omega_{\lambda}-\Omega}{\sqrt{\omega_{x}^{2}+\omega_{\perp}^{2}}}, \quad \alpha=\frac{\omega_{x}^{2} \omega_{\perp}}{\left(\omega_{x}^{2}+\omega_{\perp}^{2}\right)^{3 / 2}} .
$$

The trivial solution $y=0$ corresponds to the "spurious" excitation $\tilde{\omega}_{\lambda}=0$ in the laboratory frame with the mode $\omega_{\lambda}=\tilde{\omega}_{\lambda}+\Omega$ being a rotational mode with frequency $\Omega$ in the rotating frame. For Eq.(38) there exist three other solutions

$$
\begin{aligned}
& \omega_{n}=2 \sqrt{\frac{\omega_{x}^{2}+\omega_{\perp}^{2}}{3}} \cos \frac{\psi+2 \pi n}{3}+\Omega, \quad n=0,1,2 \\
& \cos \psi=\sqrt{27} \frac{\omega_{x}^{2} \omega_{\perp}}{\left(\omega_{x}^{2}+\omega_{\perp}^{2}\right)^{3 / 2}} \frac{r-1}{r+1}, \quad 0<\psi<\pi / 2
\end{aligned}
$$

The first solution $(n=0)$ is the one with the positive norm. Setting it equal to zero, we obtain the first bifurcation point for the negative signature 


$$
\Omega_{1}=-2 \sqrt{\frac{\omega_{x}^{2}+\omega_{\perp}^{2}}{3}} \cos \frac{\psi}{3}=\frac{\tilde{\omega}_{\lambda=-1}^{(1)}}{\lambda} .
$$

Here, the mode

$$
\tilde{\omega}_{\lambda=-1}^{(1)}=2 \sqrt{\frac{\omega_{x}^{2}+\omega_{\perp}^{2}}{3}} \cos \frac{\psi}{3}
$$

describes the quadrupole excitation in the laboratory frame, which is based on the yrast (oblate) state and carries one unit of angular momentum, i.e. $\lambda=-1$. Since $\cos \frac{\psi}{3}>\sqrt{3} / 2, \Omega_{1}$ is larger than the maximal rotational frequency (see Appendix B), it cannot be associated with a stable tilted rotation. For $\Sigma_{3}=\Sigma_{2}(\psi=\pi / 2)$ the mode $\tilde{\omega}_{\lambda=-1}^{(1)}=\sqrt{\omega_{x}^{2}+\omega_{\perp}^{2}}$ is again the isoscalar quadrupole giant resonance energy in non-rotating axially deformed nuclei [26]. Notice that this mode has been introduced in the context of a BEC under the name "scissor mode" [27]. In the spherical case it is $\tilde{\omega}_{\lambda=-1}^{(1)}=\sqrt{2} \omega_{0}$ [5]. With $\psi=0$ it is $\tilde{\omega}_{\lambda=-1}^{(1)}=2 \sqrt{\left(\omega_{x}^{2}+\omega_{\perp}^{2}\right) / 3}$.

For the $\lambda=1$ excitations the secular equation is similar to Eq.(37) with $x$ replaced by $-x$. After simplification, we obtain

$$
y\left(y^{3}-y+2 \alpha c\right)=0, \quad y=\frac{\omega_{\lambda}+\Omega}{\sqrt{\omega_{x}^{2}+\omega_{\perp}^{2}}}
$$

Again, the trivial solution $y=0$ corresponds to the "spurious" excitation $\tilde{\omega}_{\lambda}=0$ in the laboratory frame with the mode $\omega_{\lambda}=\tilde{\omega}_{\lambda}-\Omega$ being a rotational mode with frequency $-\Omega$ in the rotating frame. The three other solutions of Eq.(44) are

$$
\begin{aligned}
& \omega_{\lambda=+1}^{(1,2)}=\sqrt{\frac{\omega_{x}^{2}+\omega_{\perp}^{2}}{3}}\left(\cos \frac{\psi}{3} \mp \sqrt{3} \sin \frac{\psi}{3}\right)-\Omega \\
& \omega_{\lambda=+1}^{(3)}=-2 \sqrt{\frac{\omega_{x}^{2}+\omega_{\perp}^{2}}{3}} \cos \frac{\psi}{3}-\Omega .
\end{aligned}
$$

The positive norm solutions are the first and the second. Setting $\omega_{\lambda=+1}^{(1,2)}$ equal to zero, we obtain the bifurcation points

$$
\begin{aligned}
& \Omega_{2}=\sqrt{\frac{\omega_{x}^{2}+\omega_{\perp}^{2}}{3}}\left(\cos \frac{\psi}{3}-\sqrt{3} \sin \frac{\psi}{3}\right)=\frac{\tilde{\omega}_{\lambda=+1}^{(1)}}{\lambda} \\
& \Omega_{3}=\sqrt{\frac{\omega_{x}^{2}+\omega_{\perp}^{2}}{3}}\left(\cos \frac{\psi}{3}+\sqrt{3} \sin \frac{\psi}{3}\right)=\frac{\tilde{\omega}_{\lambda=+1}^{(2)}}{\lambda} \\
& \tilde{\omega}_{\lambda=+1}^{(1,2)}=\sqrt{\frac{\omega_{x}^{2}+\omega_{\perp}^{2}}{3}}\left(\cos \frac{\psi}{3} \mp \sqrt{3} \sin \frac{\psi}{3}\right) .
\end{aligned}
$$

Thus, they are related to the vibrational modes $\tilde{\omega}_{\lambda=+1}^{(1,2)}$ in the laboratory frame and carry $\lambda=+1$ units of angular momentum.

In fact, the lowest mode $\omega_{\lambda=+1}^{(1)}(\Omega)$ corresponds to a wobbling excitation in the intrinsic frame. As soon as the rotational frequency approaches the lowest value $\Omega_{2}$, the mode $\omega_{\lambda=+1}^{(1)}=\tilde{\omega}_{\lambda=+1}^{(1)}-\Omega$ becomes soft and signals the onset of genuine tilted rotation of the nucleus. The mode energy vanishes for

$$
\Omega_{2}=2 \sqrt{\frac{\omega_{x}^{2}+\omega_{\perp}^{2}}{3}} \cos \frac{\psi+\pi}{3}=\Omega_{\mathrm{crit}}^{(2)} .
$$

Thus, the bifurcation frequency $\Omega_{2}$ corresponds to the transition from dynamical fluctuations of the angular momentum in the oblate system to the stable tilted rotation of the triaxial shape. This critical frequency coincides perfectly with our numerical results.

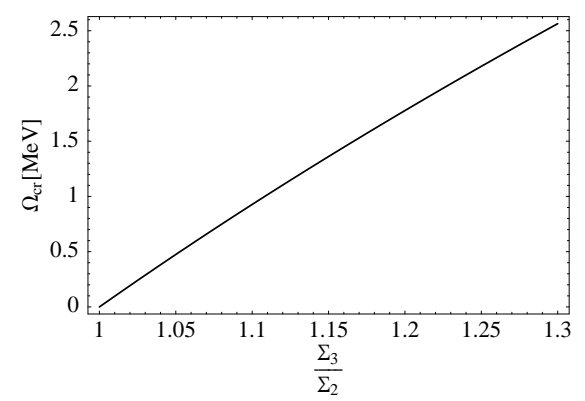

FIG. 2. Critical frequency $\Omega_{\text {crit }}^{(2)}$ versus $r=\Sigma_{3} / \Sigma_{2}$ for a typical (near) oblate situation. The example refers to $N=16$ with $\left(\Sigma_{1}, \Sigma_{2}, \Sigma_{3}\right)=(30,40,44)$; with $\omega_{x}=20.7, \omega_{\perp}=14.5$ it is $\Omega_{\text {crit }}^{(2)}=0.93$ for $r=1.1$.

Furthermore, Eq.(50) explains why oblate nuclei (at $\Omega=0$ ) cannot make the transition to tilted rotation. In fact, for these nuclei $r=1$ and hence $\psi=\pi / 2$ in Eq.(41). As a consequence, $\Omega_{\text {crit }}^{(2)}$ is zero $\left(\tilde{\omega}_{\lambda=+1}^{(1)}=0\right)$, 
i.e. no transition can occur with our convention $\Sigma_{1}<$ $\Sigma_{2}=\Sigma_{3}$. In contrast, whenever $r>1$, a positive value is obtained for $\Omega_{\text {crit }}^{(2)}$ as illustrated in Fig.2.

With $r=1$, i.e. $L_{x}=\Sigma_{3}-\Sigma_{2}=0$, the other two modes $\lambda= \pm 1$ are degenerate, since $\tilde{\omega}_{\lambda=+1}^{(2)}=\sqrt{\omega_{x}^{2}+\omega_{\perp}^{2}}=$ $\tilde{\omega}_{\lambda=-1}^{(1)}$. The corresponding critical frequencies

$$
\Omega_{\mathrm{cr}}=\tilde{\omega}_{\lambda= \pm 1} / \lambda= \pm \sqrt{\omega_{x}^{2}+\omega_{\perp}^{2}}
$$

are larger than the maximal frequency (see Appendix B) and do not give rise to symmetry breaking of the mean field. Nevertheless, these critical frequencies can be related to vibrational excitations as discussed in the following.

As was suggested by Marshalek and Sabato 28] the self-consistent cranking model should allow the study of vibrational states in spherical nuclei rotating around the symmetry axis. Our results for quadrupole excitations demonstrate that this idea can be succesfully employed for any axially deformed system rotating around the symmetry axis (see also a general discussion in [21]). The vibrational excitations with nonzero angular momentum $\lambda($ or $K$ ) aligned along the symmetry axis are determined by the bifurcation points of the mean field via Eq.(9)

$$
\tilde{\omega}_{\lambda}=\lambda \Omega_{\mathrm{cr}}
$$

The lowest critical rotational frequency (bifurcation point) gives the collective excitation with the largest angular momentum along the symmetry axis. If the critical rotational frequency lies below the maximal rotational frequency, it will give rise to a mean field symmetry breaking, as the corresponding vibrational excitation vanishes in the rotating frame. If the frequencies lie above the maximal value, they yield the higher vibrational excitation energies with nonzero angular momentum in the laboratory frame. They persist as their counterparts in the rotating frame do not vanish.

We have established numerically in all cases of triaxial oblate nuclei the onset of tilted rotation and identified exactly the value of Eq.(50) as the lowest rotational frequency. The values of $\Omega_{\text {crit }}^{(2)}$ are all fairly small in these cases $\left(\Omega_{\text {crit }}^{(2)}<1\right)$, since the values for $r$ are close to unity. This is in contrast to light prolate nuclei to which we turn next. For prolate nuclei (at $\Omega=0$ ) we have $\Sigma_{1}=\Sigma_{2} \neq \Sigma_{3}$ and the oscillator frequencies are $\omega_{x}^{0}=\omega_{y}^{0}=\omega_{\perp}^{0}$ implying the symmetry axis to be the $z$-axis. Since in this case $r>1$, Fig.2 cannot serve as a basis to exclude tilted rotation. Yet we present an independent argument.

\section{Critical frequencies for prolate rotation}

Consider a rotation around the symmetry axis, i.e. the $z$-axis. The eigenmodes have a particular simple form 15-17 and Eq.(8) leads to the nontrivial solution which must satisfy the equation

$$
\left(\omega_{+} \omega_{-}-\Omega^{2}\right)=0
$$

Setting $\omega_{x}=\omega_{y}=\omega_{\perp}^{0}$ we obtain the bifurcation point 29

$$
\Omega_{\mathrm{cr}}=\frac{\omega_{\perp}^{0}}{\sqrt{2}} .
$$

For $\Omega>\Omega_{\text {cr }}$ the axial symmetry is broken and the system is driven into the domain of triaxial shape under the $\mathrm{PAC}$ rotation. This situation is reminiscent of a striking feature established experimentally in the rotating BEC 30 32; there the minimal rotational frequency for nucleation of a vortex occurs at around $0.7 \omega_{\perp}$, where $\omega_{\perp}$ is the mean oscillation frequency of atoms in the $x-y$ 
plane, irrespective of the number of atoms or oscillation frequency $\omega_{z}$ along the $z$-axis. According to our analysis, the dynamical instability in a nuclear system and in the rotating $\mathrm{BEC}$ is of a similar nature, in spite of the different character of the interaction between nucleons (attractive) and between atoms (repulsive). The important aspect is the trapping of either system by the harmonic oscillator potential. For the nuclear system it is the mean field and for the BEC it is the external magnetic field.

For a prolate system the projection $K$ of angular momentum is a good quantum number as $\left[L_{z}, O_{K}^{\dagger}\right]=K O_{K}^{\dagger}$. We thus obtain an equation similar to Eq.(12) after replacing $\lambda$ by $K$. At the rotational frequency $\Omega_{\text {crit }}=$ $\tilde{\omega}_{K} / K$ one of the RPA frequency vanishes. For a prolate rotation the RPA equations are solved separately for each quadrupole mode, since there is no coupling between different $K$ modes.

For the quadrupole phonons with the largest projection $K=2$ it is

$$
\omega_{K=2}=\sqrt{2} \omega_{\perp}-2 \Omega
$$

This mode is the quadrupole excitation having two more units of angular momentum than the vacuum state. When $\omega_{K=2}=0$ the transition from non-collective rotation (around the $z$-axis) to triaxial collective rotation takes place, i.e. at

$$
\Omega_{\mathrm{cr}}=\frac{\omega_{\perp}^{0}}{\sqrt{2}}
$$

which is just the bifurcation point of the mean field in Eq.(54). This bifurcation point applies for any axially symmetric system (prolate and oblate) as follows from Eqs.(33) and (56).

For the $K=1$ mode the RPA solution is

$$
\omega_{K=1}=\sqrt{\omega_{\perp}^{2}+\omega_{z}^{2}}-\Omega
$$

The condition $\omega_{K=1}=0$ yields the critical frequency at which the onset of the tilted rotation should occur for the prolate system, i.e. at

$$
\Omega_{\mathrm{cr}}=\sqrt{\omega_{\perp}^{2}+\omega_{z}^{2}}
$$

However, the energy to create this mode of angular momentum $1 \hbar$ is too high, the system rather prefers the PAC rotation around the axis perpendicular to the symmetry axis. Besides, as discused above, the value of the rotational frequency in Eq.(58) lies outside the physical range of rotational frequencies (see Appendix B). As a consequence, our model does not allow tilted rotations for systems which are prolate at $\Omega=0$, i.e. if $\Sigma_{1}=\Sigma_{2}$. In contrast to the rotational frequency defined by Eqs. 33 , (56), this frequency is related to vibrational excitations carrying one unit of angular momentum in an axially symmetric system, be it oblate (Eq.(51)) or prolate (Eq. 58)).

Note that the assumption $\Sigma_{1}=\Sigma_{2}$ is essential for the arguments presented here. In contrast, if all three values of $\Sigma_{i}$ differ from each other, tilted rotations do occur at the value given by Eq.(50) just as it has been found for the near oblate situations. We recall, however, that this happens for $N<56$ at values so high that the corresponding energy minimum is only local. In contrast, when $N>70$, the relevant value of $\Omega_{\text {crit }}^{(2)}$ is sufficiently small so as yield a global minimum.

\section{SUMMARY}

Within the model considered only nuclei being triaxial in their ground state exhibit tilted rotation at a specific rotational frequency. In fact, the analytic expressions for these critical frequencies obtained in the present paper 
are in a perfect agreement with our numerical findings. All nuclei attain at $\Omega_{\text {crit }}^{(1)}$ an oblate shape. The value of $\Omega_{\text {crit }}^{(1)}$ depends on the particular nucleus. For light and medium prolate or near prolate nuclei $(N<56)$ this energy minimum is a local minimum, while for oblate or near oblate nuclei and heavy (near) prolate $(N>70)$ it is a global minimum. All triaxial nuclei - these are the nuclei which form the little 'bubbles' in Fig.1 - go through a second transition and admit a tilted rotation beyond $\Omega_{\text {crit }}^{(2)}$ while the tilted rotation cannot occur when starting with a nucleus that is axially symmetric at $\Omega=$ 0. From our model, the occurrence and identification of a tilted rotation must be seen as a signature for triaxiality. It is true that the harmonic oscillator model is a simplistic approximation for the phenomenon of the tilted rotation. However, our findings may provide guidance for values of particle number and rotational frequency at which tilted rotations may be found.

Using the cranking approach to describe non-collective rotation around a symmetry axis we have established the correspondence between symmetry-breaking bifurcation points of the mean field and the RPA frequencies carrying non-vanishing angular momentum aligned along the symmetry axis. We have demonstrated that the dynamical instability in a nuclear system and in the rotating BEC are of a similar nature, in spite of the different characters of the interaction between nucleons (attractive) and between atoms (repulsive). The remarkable agreement with regard to the bifurcation points in both systems is due to the common nature of the confining potential and its dependence on the rotational speed.
R.G.N. acknowledges financial support from the National Research Foundation of South Africa which was provided under the auspices of the Russian/South African Agreement on Science and Technology. He is also thankful for the warm hospitality which he received from the Department of Physics during his visit to South Africa. This project has been supported in part by the RFBR under the Grant 00-02-17194.

\section{APPENDIX A: EIGENMODES}

The Hamilton function of Eq.(1) can be written in matrix form

$$
H=\{\vec{p}, \vec{r}\}^{T} \mathcal{H}\{\vec{p}, \vec{r}\}
$$

where $\vec{p}$ and $\vec{r}$ are combined to the six dimensional column vector $\{\vec{p}, \vec{r}\}$ and

$\mathcal{H}=\left(\begin{array}{cccccc}1 & 0 & 0 & 0 & -\Omega_{z} & \Omega_{y} \\ 0 & 1 & 0 & \Omega_{z} & 0 & -\Omega_{x} \\ 0 & 0 & 1 & -\Omega_{y} & \Omega_{x} & 0 \\ 0 & \Omega_{z} & -\Omega_{y} & \omega_{x}^{2} & 0 & 0 \\ -\Omega_{z} & 0 & \Omega_{x} & 0 & \omega_{y}^{2} & 0 \\ \Omega_{y} & -\Omega_{x} & 0 & 0 & 0 & \omega_{z}^{2}\end{array}\right)$.

We aim at the quantum mechanical form in terms of boson operators

$$
H=\left\{Q, Q^{\dagger}\right\}^{T} \mathcal{H}_{q m}\left\{Q, Q^{\dagger}\right\}=\sum E_{j}\left(Q_{j}^{\dagger} Q_{j}+1 / 2\right)
$$

where we denote by $\left\{Q, Q^{\dagger}\right\}$ a column vector which is the transpose of the vector $\left(Q_{1}, Q_{2}, Q_{3}, Q_{3}^{\dagger}, Q_{2}^{\dagger}, Q_{1}^{\dagger}\right)$ and where

$$
\mathcal{H}_{q m}=\frac{1}{2}\left(\begin{array}{cccccc}
0 & 0 & 0 & 0 & 0 & E_{1} \\
0 & 0 & 0 & 0 & E_{2} & 0 \\
0 & 0 & 0 & E_{3} & 0 & 0 \\
0 & 0 & E_{3} & 0 & 0 & 0 \\
0 & E_{2} & 0 & 0 & 0 & 0 \\
E_{1} & 0 & 0 & 0 & 0 & 0
\end{array}\right) .
$$

It is instructive to obtain the eigenmode energies from a purely classical calculation. This approach also pro- 
vides the linear transformation between the $Q_{j}$ and the $\left\{p_{i}, x_{i}\right\}$.

The classical equations of motion for the Cartesian components of the momentum and position coordinates read

$$
\frac{d}{d t}\left(\begin{array}{l}
\vec{p} \\
\vec{r}
\end{array}\right)=\mathcal{M}\left(\begin{array}{l}
\vec{p} \\
\vec{r}
\end{array}\right)
$$

The matrix $\mathcal{M}$ is given by

$$
\mathcal{M}=\left(\begin{array}{cc}
0 & -I_{3} \\
I_{3} & 0
\end{array}\right) \mathcal{H}
$$

where $I_{3}$ is a 3 by 3 unit matrix. The classical orbits are the solution of Eq.(A.5) which reads

$$
\{\vec{p}(t), \vec{r}(t)\}=\mathcal{U} \exp (\mathcal{D} t) \mathcal{V}\{\vec{p}(0), \vec{r}(0)\}
$$

where $\mathcal{D}=\operatorname{diag}\left(-i E_{1},-i E_{2},-i E_{3}, i E_{3}, i E_{2}, i E_{1}\right)$ is the diagonal form of $\mathcal{M}$. The initial conditions $\{\vec{p}(0), \vec{r}(0)\}$ are of no interest here. From Eq. A.6) it follows that the eigenmodes listed in Eq.(A.4) occur in the classical problem in this form. They are obtained from the secular equation

$$
\operatorname{det}\left|E I_{6}-\mathcal{M}\right|=0
$$

The above equation turns out to be a third order polynomial in $E^{2}$ and has also been found by 14. The column vectors of $\mathcal{U}$ are the (complex) right hand eigenvectors of $\mathcal{M}$. Note that $\mathcal{M}$ is not symmetric, hence neither $\mathcal{U}$ nor $\mathcal{V}$ are unitary, yet $\mathcal{V}=\mathcal{U}^{-1}$. We denote the column vectors of $\mathcal{U}$ by $u^{(k)}$, they obey the equations

$$
\begin{aligned}
\left(\mathcal{M}+i E_{k} I\right) u^{(k)} & =0, \quad k=1,2,3 \\
\left(\mathcal{M}-i E_{7-k} I\right) u^{(k)} & =0, \quad k=4,5,6
\end{aligned}
$$

which can be solved as an inhomogeneous system by choosing an arbitrary component of $u^{(k)}$ equal to unity. The proper normalisation is achieved by the observation that, up to normalisation factors, the matrix $\mathcal{V}=\mathcal{U}^{-1}$ can be written as

$$
\mathcal{V}=\left(\begin{array}{cccccc}
0 & 0 & 0 & 0 & 0 & -i \\
0 & 0 & 0 & 0 & -i & 0 \\
0 & 0 & 0 & -i & 0 & 0 \\
0 & 0 & i & 0 & 0 & 0 \\
0 & i & 0 & 0 & 0 & 0 \\
i & 0 & 0 & 0 & 0 & 0
\end{array}\right) \mathcal{U}^{T}\left(\begin{array}{cc}
0 & -I_{3} \\
I_{3} & 0
\end{array}\right)
$$

This implies that $\mathcal{U}^{T} \mathcal{H U}$ is in fact skew-diagonal as in Eq. A.4), and therefore $\mathcal{U}$ can be normalised such that $\mathcal{U}^{T} \mathcal{H U}=\mathcal{H}_{q m}$. This implies in particular

$$
\{\vec{p}, \vec{r}\}=\mathcal{U}\left\{Q, Q^{\dagger}\right\}
$$

The properties of the matrix $\mathcal{U}$ guarantee the commutator $\left[Q_{k}, Q_{k^{\prime}}^{\dagger}\right]=\delta_{k, k^{\prime}}$ as a consequence of $\left[x_{i}, p_{j}\right]=$ $i \hbar \delta_{i, j}$. Clearly, for $\Omega=0$ the $Q_{j}$ become the usual operators $a_{i}=\left(p_{i}-i m \omega_{i} x_{i}\right) / \sqrt{2 m \hbar \omega_{i}}$.

\section{APPENDIX B: MAXIMAL ROTATIONAL FREQUENCY}

At particular values of $\Omega$ the roots of cubic equation become negative, i.e., the corresponding eigenmodes $E_{k}$ would be imaginary. Let us consider the simple case of a rotation about the principal axis $x$. The solutions for the eigenmodes are well known 15 17

$$
E_{1}=\omega_{x} ; \quad E_{2}=\omega_{+} ; \quad E_{3}=\omega_{-}
$$

$\omega_{ \pm}^{2}=\frac{\omega_{y}^{2}+\omega_{z}^{2}}{2}+\Omega^{2} \pm \frac{1}{2}\left[\left(\omega_{y}^{2}-\omega_{z}^{2}\right)^{2}+8 \Omega^{2}\left(\omega_{y}^{2}+\omega_{z}^{2}\right)\right]^{1 / 2}$

Thus, for the eigenmodes to be real, $\omega_{y}, \omega_{z}$ and $\Omega$ must satisfy

$$
\frac{\omega_{y}^{2}+\omega_{z}^{2}}{2}+\Omega^{2} \geq \frac{1}{2}\left[\left(\omega_{y}^{2}-\omega_{z}^{2}\right)^{2}+8 \Omega^{2}\left(\omega_{y}^{2}+\omega_{z}^{2}\right)\right]^{1 / 2}
$$


which leads to the inequality

$$
\left(\Omega^{2}-\omega_{y}^{2}\right)\left(\Omega^{2}-\omega_{z}^{2}\right) \geq 0 .
$$

This means that the frequency $\Omega$ must be either larger or smaller than both frequencies. Since the condition $\Omega \geq \sup \left(\omega_{y}, \omega_{z}\right)$ is unphysical, the rotational frequency should not exceed the maximal rotational frequency $\Omega_{\max } \leq \inf \left(\omega_{y}, \omega_{z}\right)$.

\section{APPENDIX C: QUADRUPOLE OPERATORS}

For a solution of the RPA equation of motion it is convenient to use the following representation of the quadrupole operators

$$
\begin{aligned}
& D_{0}=\sqrt{\frac{5}{16 \pi}}\left(2 \bar{z}^{2}-\bar{x}^{2}-\bar{y}^{2}\right) \\
& D_{1}=\frac{i}{\sqrt{2}}\left(D_{21}+D_{2-1}\right)=\sqrt{\frac{15}{4 \pi}} \bar{y} \bar{z} \\
& D_{2}=\frac{1}{\sqrt{2}}\left(D_{22}+D_{2-2}\right)=\sqrt{\frac{15}{16 \pi}}\left(\bar{x}^{2}-\bar{y}^{2}\right) \\
& D_{3}=\frac{1}{\sqrt{2}}\left(D_{21}-D_{2-1}\right)=-\sqrt{\frac{15}{4 \pi}} \bar{x} \bar{z} \\
& D_{4}=\frac{i}{\sqrt{2}}\left(D_{22}-D_{2-2}\right)=-\sqrt{\frac{15}{4 \pi}} \bar{x} \bar{y}
\end{aligned}
$$

With these definitions we have $\sum_{\mu=-2}^{2} D_{\mu}^{\dagger} D_{\mu} \equiv$ $\sum_{m=0}^{4} D_{m}^{2}$. Using the transformation Eq. A.11) the quadrupole operators are expressed in terms of the boson operators $Q_{i}, Q_{i}^{\dagger}$. In case of the one-dimensional rotation the matrix $\mathcal{U}$ reduces to the transformation as in [17] and all matrix elements are calculated analytically. When the signature is a good quantum number, the operators can be classified according to their signature quantum number: the operators $D_{0}, D_{1}, D_{2}$ are characterized by the positive, while the operators $D_{3}, D_{4}$ are characterized by the negative signature quantum number [6, 7,23]. In the oblate case it is convenient to calculate the matrix ele- ments using $x$-axis quantization, since the symmetry axis coincides with the axis of rotation.

[1] S. Frauendorf, Rev.Mod.Phys. 73, 463 (2001)

[2] S.G. Nilsson and I. Ragnarsson, Shapes and Shells in Nuclear Structure (Cambridge University Press, Cambridge, 1995)

[3] S. Frauendorf, Nucl.Phys.A 557, 259c (1993)

[4] S. Stringari, Phys.Rev.Lett. 86, 4725 (2001)

[5] A. Bohr and B.R. Mottelson, Nuclear Structure, Vol.2 (Benjamin, New York, 1975)

[6] D. Janssen and I.N. Mikhailov, Nucl.Phys. A318, 390 (1979)

[7] E.R. Marshalek, Nucl.Phys. A331, 429 (1979)

[8] I.N. Mikhailov and D. Janssen , Phys.Lett.B 72, 303 (1978)

[9] D. Janssen, I.N. Mikhailov, R.G. Nazmitdinov, B. NerloPomorska, K. Pomorski, and R.Kh. Safarov, Phys.Lett.B 79, 347 (1978)

[10] F.R. May, I.N. Mikhailov, R.G. Nazmitdinov, and D. Janssen, Sov.J.Nucl.Phys. 24, 486 (1978); D. Janssen, F.R. May, I.N. Mikhailov and R.G. Nazmitdinov, Phys.Lett.B 73, 271 (1978)

[11] H. Frisk and R. Bengtsson. Phys.Lett. B196, 14 (1987)

[12] W.D. Heiss and R.G. Nazmitdinov, Phys.Lett.B 397, 1 (1997)

[13] W.D. Heiss and R.G. Nazmitdinov, JETP Lett. 72, 106 (2000)

[14] M.G. Vasanji and M. Harvey, Nucl.Phys. A344, 61 (1980)

[15] J.G. Valatin, Proc.Roy.Soc. 238, 132 (1956)

[16] G. Ripka, J.P. Blaizot, and N. Kassis, in International Extended Seminar, Trieste, 1973 (IAEA, Vienna, 1975) Vol.1, p. 445

[17] V.G. Zelevinsky, Sov.J.Nucl.Phys. 22, 565 (1976)

[18] T. Troudet and R. Arvieu, Ann.of Phys. (NY) 134, 1 (1981)

[19] Ts. Dankova and G. Rosensteel, Phys.Rev.C 63, art.N 054303 (2001)

[20] R.P. Feynman, Phys.Rev. 56, 340 (1939)

[21] E.R. Marshalek, Phys.Rev.C 54, 159 (1996)

[22] T. Kishimoto, J.M. Moss, D.H. Youngblood, J.D. Bronson, C.M. Rozsa, D.R. Brown and A.D. Bacher, Phys.Rev.Lett. 35, 552 (1975); S. Åberg, Phys.Lett.B 157, 9 (1985); H. Sakamoto and T. Kishimoto, Nucl.Phys.A 501, 205 (1989)

[23] J. Kvasil and R.G. Nazmitdinov, Sov.J.Part.Nucl. 17, 265 (1986)

[24] A. Akbarov, A.V. Ignatyuk, I.N. Mikhailov, Kh.L. Molina, R.G. Nazmitdinov, and D. Janssen, Preprint P4-12772, JINR, Dubna, 1979; Sov.J.Nucl.Phys. 33, 794 (1981)

[25] H. Kurasawa, Progr.Theor.Phys. 64, 2055 (1980)

[26] T. Suzuku and D.J. Rowe, Nucl.Phys.A 299, 461 (1977) 
[27] D. Guery-Odelin and S. Stringari, Phys.Rev.Lett. 83, 4452 (1999)

[28] E.R. Marshalek , Phys.Rev.C 3, 1710 (1971); E.R. Marshalek and M. Sabato, ibid 4, 1006 (1971); ibid 5, 1130 (1972)

[29] E.R. Marshalek and R.G. Nazmitdinov, Phys.Lett.B 300, 199 (1993); E.R. Marshalek, R.G. Nazmitdinov and I. Ragnarsson, Bull.Russ.Acad.of Science, Physics 57, 1709 (1993)

[30] K.W. Madison, F. Chevy, W. Wohlleben, and J. Dalibard, Phys.Rev.Lett. 84, 806 (2000)

[31] F. Chevy, K.W. Madison, and J. Dalibard, Phys.Rev.Lett. 85, 2223 (2000)

[32] S. Sinha and Y. Castin, Phys.Rev.Lett. 87, 190402 (2001)

[33] O.M. Marago, S.A. Hopkins, J. Arlt, E. Hodby, G. Hechenblaikner, and C.J. Foot, Phys.Rev.Lett. 84, 2056 (2000) 